\title{
Cumulativité des connaissances et science
} économique

Que cherche-t-on exactement à cumuler?

\section{Pascal Bridel}

\section{OpenEdition}

\section{Journals}

Édition électronique

URL : http://journals.openedition.org/ress/376

DOI : $10.4000 /$ ress.376

ISSN : 1663-4446

Éditeur

Librairie Droz

Édition imprimée

Date de publication : 1 février 2005

Pagination : 63-79

ISBN : 2-600-00958-2

ISSN : 0048-8046

Référence électronique

Pascal Bridel, «Cumulativité des connaissances et science économique », Revue européenne des sciences sociales [En ligne], XLIII-131 | 2005, mis en ligne le 05 novembre 2009, consulté le 02 mai 2019. URL : http://journals.openedition.org/ress/376 ; DOI : 10.4000/ress.376 
Pascal BRIDEL

\section{CUMULATIVITÉ DES CONNAISSANCES ET SCIENCE ÉCONOMIQUE Que cherche-t-on exactement à cumuler?}

\section{INTRODUCTION}

Dans son texte introductif, Denise Pumain (2005) suggère toute une série de portes d'entrée dans un sujet qui ressemble à une superbe construction baroque, mais à une construction pleine de courants d'air et de portes qui claquent. De quelles connaissances parle-t-on? que cherche-t-on à cumuler? Doit-on (peut-on?) une fois encore faire appel à la philosophie des sciences dures pour expliquer les conditions de remplacement d'une théorie en sciences sociales par une autre? Et dans ce cadre-là, doit-on favoriser une approche relativiste basée sur une sociologie de nos diverses professions; ou, inversement, doit-on se concentrer d'une manière plus absolutiste sur la logique interne du 'progrès technique' de la théorie économique (comme branche de la science économique)? ${ }^{1}$ La cumulativité du savoir en science économique relève-t-elle d'une manière instrumentaliste de la théorie de la 'croissance endogène'? ou, au contraire, la science économique, à l'exemple de la sociologie, est-elle un savoir historique qui, pour reprendre les termes de Passeron, 'ne peut pas prendre la forme d'un savoir cumulatif, c'est-àdire d'un savoir dont un paradigme théorique organiserait les connaissances cumulées' (1991, p. 364); ou, plus vraisemblablement, tel Janus, la science économique ne relèverait-elle pas simultanément, à la Veblen/Myrdal, d'une 'cumulativité causale' reflétant le paradoxe d'une discipline des sciences humaines qui cherche désespérément à devenir une science dure mais qui, largement privée d'autre laboratoire que le savoir historique, semble avoir atteint un stade indépassable de ce rapprochement (Malinvaud, 1995)? En effet, largement privée des capacités expérimentales des sciences exactes, la science économique semble avoir atteint un stade de son développement où plus elle tente par ses méthodes de se rapprocher des sciences dures, plus cet objectif semble se dérober. Ou, en d'autres termes encore, comment juger de la cumulativité des connaissances en science écono-

Dans cet ordre d'idée, les historiens de la pensée économique ont beaucoup débattu cette question sous le vocable de 'Whig history of economics'. Ce débat est centré sur l'idée de savoir s'il convient de comprendre (de reconstruire?) le passé dans la perspective d'un schéma d'analyse contemporain. Les opposants à cette 'Whig history of economics' considèrent qu'il est indispensable de relire les théories du passé en tenant compte des préjugés, des croyances et des idéologies de ceux qui les ont conçues et qui peuvent être radicalement différentes de celles adoptées par les économistes d'aujourd'hui. Dans sa forme la plus extrême, cette approche refuse de considérer qu'il puisse y avoir cumulativité et croissance de la connaissance en science économique: il y a simplement une redéfinition des questions abordées. 
mique sans convoquer à la fois - pour faire simple - le paradigme du choix rationnel (intimement lié à l'individualisme méthodologique) et l'idée que toute action de l'agent doit être historiquement contextualisée. La science économique n'est-elle pas à la fois une discipline cumulable et une science dont le savoir contextualisé n'est pas cumulable? En bref, en science économique, la connaissance - et sa possible cumulativité - doivent être passées au double crible de la cohérence interne et externe des modèles dont elle se compose. Ou, au contraire, la rigueur de la déduction logique est-elle le seul moyen de servir de substitut à une base expérimentale défaillante parce qu'impossible? En dernière analyse, et la double signification du mot connaissance rend ce paradoxe encore plus aigu, comment juger de la cumulativité des connaissances en science économique alors que la plupart des modèles théoriques impliquent notamment pour les agents une connaissance/information parfaite, des prévisions parfaites et des anticipations rationnelles alors que, dans le monde réel, l'incertitude est probablement l'une des caractéristiques les plus évidentes de toute activité économique. Malheureusement, dès que l'on abandonne, ne serait-ce que marginalement, la matrice initiale du choix rationnel, les modèles s'effritent, la clarté des conclusions se brouille et l'avantage comparatif des économistes se dilue. Comment donc relier dans la durée et d'une manière cohérente ces deux visages de Janus?

Cette contribution va s'efforcer de réduire la dimension colossale du problème posé par l'idée de cumulativité en tentant de répondre uniquement à une toute petite partie d'une question formulée par Denise Pumain: "Comment se joue le couple formalisation-observation dans les disciplines qui ont développé un corpus formel important comme l'économie?». En particulier, on va tenter de s'interroger sur les possibles capacités cumulatives respectives de la formalisation et de l'observation pour se demander finalement comment il est possible ou non de les combiner. Pour tenter non d'atteindre mais d'approcher cet objectif ambitieux, on emprunte une démarche (due à Lawson) dans la tradition de Cambridge et basée sur l'opposition entre la nature relative et absolue du savoir en théorie économique ${ }^{2}$ (section I). Cette dichotomie est ensuite mise en relation avec les couples empirisme/rationalisme et réalisme/instrumentalisme et on tente d'en tirer quelques implications pour la science économique (section II). Finalement, la cumulativité des connaissances en science économique est montrée comme étant simultanément quantitative et qualitative, qu'elle dépend d'un interactionisme social (section III) et donc, pour paraphraser Denise Pumain, qu'elle ne peut se concevoir qu'en 'articulant explicitement des concepts issus de disciplines différentes': il s'agirait alors de déconstruire l'approche strictement disciplinaire des économistes en vue de reconstruire un autre savoir à un niveau plus englobant (2004, p. 5).

\section{CERTITUDE ET INCERTITUDE DU SAVOIR EN SCIENCE ÉCONOMIQUE}

Très classiquement, en théorie économique, une approche réaliste implique que les objets soumis à la recherche aient une existence largement indépendante

\footnotetext{
«The relative/absolute nature of knowledge and economic analysis », Lawson (1987), p. 951.
} 
de la démarche analytique dont ils sont l'objet. Un monde économique objectif existerait indépendamment de la conscience que peut en avoir le théoricien. La connaissance de ce monde objectif passe alors nécessairement par la conscience que le théoricien acquiert progressivement de cette existence. Pour l'instrumentalisme aujourd'hui largement dominant en théorie économique, les objets soumis à la recherche sont considérés comme de simples 'convenient fictions', des fictions commodes et pratiques. La distance - et non la proximité - du modèle théorique à la réalité contribue mieux que toute description pseudo réaliste à la compréhension de la logique de fonctionnement du monde économique ${ }^{3}$.

Illustrons à l'aide d'un premier exemple. Le degré d'information attribués aux agents est l'une des lignes de partage les plus importantes qui sépare ces deux approches. À l'exemple de Keynes et dans la tradition des probabilités subjectives, les réalistes considèrent l'incertitude comme l'une des caractéristiques les plus incontournables de la vie économique. Inversement, l'approche instrumentaliste (dont l'équilibre général à la Arrow-Debreu est l'épitomé) suppose volontiers que les agents sont par exemple au bénéfice d'une information parfaite, qu'ils sont 'infiniment clairvoyants' (pour utiliser l'expression de Poincaré dans une lettre à Walras ) et/ou au bénéfice d'anticipations rationnelles. Lorsque le réaliste s'intéresse à la notion d'incertitude, il s'efforce de concentrer son analyse sur des situations pour lesquelles il dispose - ou croît disposer - de connaissances probabilisables (et peut-être même quantifiables et mesurables). Par extension, cette thèse de Lawson suggère que, paradoxalement, la connaissance en science économique est à la fois certaine (et donc cumulable) et incertaine (et donc non cumulable): le problème central est donc bien de construire un pont entre une théorie pure qui, comme la théorie de l'équilibre général, relève des mathématiques appliquées et une théorie appliquée dont l'application historique doit être contextualisée ${ }^{4}$. Aussi clairement que, tôt ou tard, l'instrumentaliste doit admettre que l'idée d'une relativisation, d'une historicisation de sa théorie est indispensable à son application au 'monde réel', le relativiste doit bien aussi admettre qu'une observation quotidienne de la société révèle la présence parmi les agents qui la composent de certitudes et de prétentions à une vérité (de croyances?) qui ne sauraient être négligées 5 .

La fréquentation quotidienne de mes collègues m'a appris que beaucoup d'économistes - notamment ceux qui sont directement ou indirectement impliqués dans la conduite de la politique économique - défendent fréquemment leurs opinions comme relevant de vérités qui peuvent être considérées comme quasicertaines mais qui ne relèvent en aucun cas d'une démonstration théorique: 'La

Il s'agit bien entendu, et une fois encore, de la vieille querelle des universaux entre des universalia ante rem et des universalia post rem. En théorie économique, cette querelle remonte au moins à la dispute à propos du modèle d'équilibre général entre le réalisme de Walras et le nominalisme de Pareto.

Comme il été démontré d'une manière maintenant parfaitement convaincante, jusqu'au milieu des années 1930, et en particulier pour Walras et Pareto, les premiers théoriciens de l'équilibre général ont abondamment réfléchi sur les liens à établir entre théorie pure et théorie appliquée (Ingrao et Israel, 1990; Baranzini et Bridel, 2004).

La question lancinante de savoir si l'on peut fonder microéconomiquement la valeur positive de la monnaie est un autre exemple contemporain de cette opposition (Bridel, 2002). 
théorie économique nous dit que...'; 'l'inflation est partout et toujours un phénomène monétaire...'; dans le long terme, la monnaie est neutre...; 'les déficits budgétaires sont résorbés par la croissance qu'ils provoquent...; etc. Frank Hahn écrivait en 1985 à propos des monétaristes et des Friedmaniens alors au zénith de leur influence sur la profession d'économiste:

... the display of certainty by many economists in discussing matters of economic policy is not only lacking in honesty but extremely harmful to the subject (1985, p. 27).

Il est évident que dans bien des occasions, de tels arguments d'autorité, ou une invocation à de pseudo certitudes ne sont rien d'autres que des artifices rhétoriques ou une manière d'intimider ses opposants (ou ses maîtres politiques). Et pourtant... Peut-on vraiment toujours attribuer de telles démarches (si fréquentes !) exclusivement à une forme de malhonnêteté intellectuelle? Lorsque dans sa biographie intellectuelle (intitulée In praise of the market), Brunner nous fait part de son admiration quasi-religieuse pour le principe de coordination marchande ou que Friedman parle en termes messianiques de son projet monétaire (dont aucun modèle théorique connu ne peut soutenir les conclusions), ne doit-on pas sérieusement s'interroger sur l'influence des 'croyances certaines' en science économique $^{6}$. Inversement, lorsque Bourdieu s'exprime sur la 'théorie économique' mathématique, il fait preuve d'une croyance non moins certaine lorsqu'il affirme que celle-ci 'consiste à mettre entre parenthèses les conditions économiques et sociales des dispositions rationnelles ... et des structures économiques et sociales qui sont la condition de leur exercice' (1998, p. 109). En bref, et pour reprendre la vieille distinction de Pareto, le théoricien en sciences sociales est doté d'une remarquable capacité à déguiser une approche non logique en théories logiques pouvant s'insérer dans un modèle analytique qui leur donnera une apparence de 'vérité scientifique'. Et le rôle de ces 'croyances certaines' en science économique ne peut pas et ne doit pas être négligé. Axer exclusivement son analyse sur l'incertitude (sous prétexte qu'elle est omniprésente) revient alors à négliger que la certitude (des croyances?) est aussi une caractéristique omniprésente de la vie économique.

Dans les deux cas, la réconciliation entre formalisation et observation (même stylisée) est cruciale pour juger des capacités cumulatives de la science économique. Dans le cadre de l'épistémologie traditionnelle, on renverra volontiers ces deux approches à l'empiricisme et au rationalisme. Dans le cadre de l'acquisition du savoir, le premier met principalement l'accent sur l'expérience sensorielle (savoir relatif); le second donne la priorité au raisonnement a priori (savoir absolu). Une fois encore, et si l'on reprend la tradition cambridgienne issue du Treatise on Probability (1921) de Keynes, les deux éléments sont indissociables. En d'autres termes, il ne semble pas y avoir une frontière parfaitement claire (pour reprendre notre exemple précédent) entre certitude et incertitude, entre savoir relatif et savoir absolu. Dans les propres termes de Keynes:

That part of our knowledge which we obtain directly, supplies the premisses of that part which we obtain by argument. From these premisses we seek to justify some degree of rational belief about all sorts of conclusions (1921, p. 121).

\footnotetext{
- Une telle certitude n'est de loin pas exclusivement le fait des économistes...
} 
Même si, entre empiricisme et rationalisme, la position de Keynes est souvent pour le moins ambiguë, la connaissance directe est pour lui le fondement de toute connaissance. En bref, il n'existe pas de connaissance pure d'un point de vue absolu qui ne puisse être remplacée par une meilleure approximation de la réalité. Aucune connaissance ne saurait être toutefois dans l'erreur complète?

\section{IMPLICATIONS POUR LA CONNAISSANCE EN SCIENCE ÉCONOMIQUE}

Paraphrasant la démarche qui vient d'être proposée, il est possible d'affirmer que toute action économique est déterminée par une expérience et un savoir contextualisé; toute action de l'agent économique est donc reliée à un environnement incluant par exemple ses expériences, ses croyances et bien sûr un type particulier de rationalité. La connaissance en science économique a donc bien un aspect relatif. Cela ne signifie pourtant pas que toute connaissance en science économique n'est que relative. Cela signifie simplement que la dimension relative de la connaissance ne peut pas être négligée. Et c'est à ce niveau précis que, dans le domaine de la théorie économique la plus abstraite (équilibre général ou théorie des jeux), on se heurte à une absence presque totale de cette dimension relative du savoir. Y a-t-il une quelconque signification, et dans quelle mesure, à construire une théorie économique de ce type? En d'autres termes, et l'on rejoint le rapport formalisme-observation de Denise Pumain, si la théorie économique veut être d'une quelconque utilité, si le théoricien cherche à comprendre et à analyser des formes existantes de rationalité, à déchiffrer des actions observables des agents, et, finalement à influencer l'activité des agents économiques par le biais de politiques économiques, il est évident que, dans une certaine mesure il ne peut se contenter de raisonnements a priori: il doit 'mettre les mains dans le cambouis', 'mouiller sa chemise' et se 'frotter à la réalité'. Si, comme on va le voir, la théorie économique semble largement exclure la relativité du savoir et toute contextualisation, on pourrait alors s'interroger à juste titre sur la nature, et l'utilité, de ce savoir. De plus, depuis une quinzaine d'années, cette tendance la rend extrêmement vulnérable à la critique postmoderniste qui met sérieusement en doute l'idée de progrès, et donc de cumulativité, en théorie économique. En refusant de reconnaître qu'il puisse exister des règles qui gouvernent la croissance de la connaissance en économie, ce courant considère alternativement la théorie économique comme une simple rhétorique ou, une fois encore, comme une manière sophistiquée de parler politique. De par sa sophistication instrumentale, la théorie économique est évidemment une victime de choix pour 'l'égalitarisme cognitif' (Laudan, 1984, p. 30) dont souffre aujourd'hui tout discours scientifique. Il ne faut toutefois pas confondre cette critique post-moderniste (il n'y a pas de règles...) avec une critique plus subtile: il y a bien des règles qui gouverne la croissance de la connaissance, mais la science économique ne les respecterait simplement pas. Mais est-ce bien le cas?

Même si après Einstein, la physique de Newton n'a plus été considérée comme 'vraie', auparavant elle était bien considérée comme 'vraie'. La physique de Newton est donc bien une connaissance absolue et relative. 
Dans une perspective réaliste d'une lecture relativiste/absolutiste de la connaissance, une analyse économique qui présuppose des fondements théoriques immuables (la théorie du choix rationnel, par exemple) doit 1) soit aboutir à des conclusions sans rapport (même lointain) avec une quelconque réalité (même stylisée) 2) soit s'intéresser à un objectif explicitement différent du réalisme du modèle. On rejoint ici l'opposition mentionnée plus haut entre empirisme et rationalisme. $\mathrm{Ou}$, pour reprendre un vieux débat méthodologique entre économistes des années cinquante, l'opposition entre measurement without theory et theory without measurement.

En science économique, la tribu des 'empiristes naïfs' est aujourd'hui une espèce en voie de disparition. Peu d'économistes ${ }^{8}$ sont enclins à affirmer la primauté de la collecte objective de données qui représenteraient des faits bruts non contaminés par des présupposés, des jugements de valeur ou une quelconque théorie. Facts are theory laden et la connaissance ne saurait résulter d'un simple examen de données brutes: facts don't speak for themselves écrit Thomas Sargent (2002, p. 7). Une grille analytique aussi sommaire soit-elle est indispensable à n'importe quelle collecte de 'faits'.

Certes à des degrés différents, l'immense majorité des économistes donne aujourd'hui un rôle prépondérant - si ce n'est exclusif - au raisonnement a priori. Héritière du bourbakisme, l'axiomatique à la Debreu en est évidemment le cas le plus extrême. Une telle approche peut difficilement satisfaire une vision réaliste ${ }^{9}$ de l'analyse économique qui donnerait une priorité à une interaction continue entre la théorie, l'expérimentation, l'observation et la pratique de la politique économique. Dans un passage célèbre de l'introduction de sa Théorie de la valeur (1966), Debreu affirme qu'une structure théorique axiomatique doit nécessairement 1) être construite sans se réclamer d'une quelconque réalité et, 2) bien sûr, sans suggérer une quelconque interprétation que l'on pourrait lui donner par la suite. Dans les termes même de Debreu:

La fidélité à la rigueur dicte la forme axiomatique de l'analyse où la théorie, au sens strict, est logiquement disjointe de ses interprétations. ... Une telle dichotomie révèle toutes les hypothèses de la structure logique de l'analyse. Elle rend aussi possible des extensions immédiates [cumulatives?] de cette analyse sans modification de la théorie par simple réinterprétation des concepts... (1966, p. XLIV)

En fait, pour Debreu, la structure axiomatique de sa théorie de l'équilibre général ne représente ni un modèle ni une abstraction de la réalité économique et il n'existe aucune nécessité de la confronter à des observations empiriques. La rigueur de la déduction logique, on le verra, doit servir de substitut à une base expérimentale défaillante parce qu'impossible. L'approche axiomatique donne naissance à une structure formelle que l'on doit analyser pour elle-même, indépendamment de toute interprétation que l'on pourrait lui donner ou pas. Parler d'irréalisme à propos du modèle Arrow-Debreu n'a aucun sens. Ou, pour paraphraser Weintraub (1985, p. 119), se demander si le modèle Arrow-Debreu est réfutable n'est pas faire preuve d'un positivisme de bon aloi ou même d'une

\footnotetext{
$\therefore$ Les exemples explicites et avoués sont rares et anciens (voir Hempel, 1966, p. 11).

' Avec Mäki, on devrait plutôt parler, de 'réalisticité'.
} 
certaine rigueur scientifique. Au contraire! Poser une telle question démontre simplement la confusion intellectuelle de celui qui la formule. Toute théorie axiomatique ne saurait être soumise à d'autre test de réfutabilité que celui offert par sa cohérence interne. Une certaine cumulativité semble dès lors envisageable.

Au-delà du stricte purisme axiomatique de Debreu, la plupart des théoriciens de l'équilibre général (MacKenzie, Arrow et Hahn pour ne mentionner que les plus célèbres), et peut-être parce qu'ils ne sont pas des Normaliens bourbakistes, ne réclament pas explicitement que les axiomes économiques à la base de l'équilibre général soient choisis complètement indépendamment de leur interprétation. Même dans un cadre axiomatique, certaines métarègles du jeu sont implicitement postulées: l'hypothèse de survie du consommateur, l'idée de rendements d'échelle modérément croissants, l'impossibilité pour les agents de faire faillite, ou, plus générale encore, l'idée que l'échange entre deux agents doit être librement consenti pour garantir une amélioration au sens de Pareto ou encore que l'on ne peut pas passer son temps à assassiner ses coéchangistes ou à les réduire en esclavage même si de telles actions devaient permettre de maximiser son utilité totale.

Courant le risque d'être taxé d'aprioriste et de nager en plein relativisme historique, Hahn semble ainsi disposer à défendre l'idée que les axiomes à la base de l'équilibre général ont en fait un rapport avec le monde 'réel':

Axioms are not plucked out of thin air, and far from distancing the theorists from what somewhat mysteriously is called the 'real' world, they constitute claims about this world so widely agreed as to make further argument unnecessary (1985, p. 12).

N'a-t-on pas là des 'croyances certaines', une connaissance contextualisée du monde réel qui viennent s'immiscer dans les axiomes d'une démarche analytique qui se veut purement rationnelle et absolutiste? N'a-t-on pas affaire à une connaissance qui est à la fois relative et absolue? Le problème avec ces 'axiomes qui sont largement acceptés sans discussion' est bien évidemment qu'ils sont sans contenu analytique s'ils ne sont pas, au préalable, complètement retravaillé et complété par des hypothèses complémentaires qui font, elles, naturellement problème. Prenons par exemple l'affirmation banale et difficilement contestable que 'les agents ont des préférences'. Pour passer de cette banale constatation constamment vérifiée dans la vie quotidienne à une théorie du choix rationnel, il faut ajouter à cette 'croyance certaine' tout un arsenal analytique pour obtenir une fonction d'utilité croissante, continue, deux fois différentiable et strictement quasi-concave reflétant la complétude, la réflexivité et la transitivité d'un ordre de préférence individuel. Hahn commente prudemment:

However, at the end we shall have to agree that the genuine axiom, persons have some preferences, has been idealised and strengthened by theorists beyond the point at which it commands universal consent (1985, p. 14).

Cette 'idéalisation et ce renforcement' marque bien le passage d'une connaissance relative à une connaissance absolue. Cette démarche, qui rappelle bien sûr en théorie économique le passage que fait Walras du type réel du marché de la bourse au type idéal de la libre concurrence absolue, implique de multiples hypothèses 'de convenance' essentiellement faites pour des motifs de 'tractabilité 
mathématique' ${ }^{10}$. Et ce sont ces hypothèses de convenance qui permettent de construire une structure axiomatique qui devient alors arbitraire, aprioriste ${ }^{11}$, qui perd tout contact avec une quelconque réalité et qui, par là, devient un 'paradigme théorique' permettant une cumulativité du savoir. L'histoire analytique de l'équilibre général (par opposition à l'histoire de l'usage que l'on fait de ce modèle) démontre bien la cumulativité du savoir acquis pour déterminer, par exemple, les conditions nécessaires et suffisantes à la définition d'un vecteur de prix d'équilibre, et donc, à la définition d'un théorème d'existence d'un équilibre général ${ }^{12}$.

Dans un de ses nombreux plaidoyers en faveur de l'équilibre général Hahn reformule d'une manière légèrement différente la position initiale de Debreu en affirmant qu'il est impossible d'accepter le caractère heuristique de l'équilibre général et de, simultanément, le considérer comme une description même abstraite de la réalité. En effet, la théorie de l'équilibre général peut aussi bien s'appliquer à une économie de marché qu'à une économie centralisée, à une économie de troc qu'à une économie monétarisée. De plus, comme le reconnaît explicitement Arrow, cette théorie pure des marchés n'est en théorie, compatible avec aucun régime politique, aucune forme de gouvernement, ni la démocratie, ni l'oligarchie, ni la dictature. Et pourtant, inutile de dire que beaucoup d'économistes considère ce modèle comme descriptif et normatif. Les vrais professionnels de la théorie eux affirment tous explicitement, comme Hahn, que 'the aim is not realism...' (1985, p. 13), mais quelque chose d'autre. Oui, mais quoi au juste au-delà de l'exercice de mathématique appliquée? Le savons-nous vraiment?

Finalement, du point de vue du réaliste qui cherche à construire une démarche dont l'objet est d'accroître d'une manière cumulative notre connaissance du monde économique dynamique dans lequel nous vivons, la science économique semble tourner en rond (au moins depuis Ricardo!). Les explications empiriques (du type école historique allemande) qui donne un rôle central à la collecte de faits et de données libres de jugements de valeur et de toute grille interprétative sont sans issue. De même, les explications rationalistes mettant en avant le rôle exclusif et central du raisonnement a priori utilisent par la force des choses des critères de validité/justification qui n'ont rien à voir avec un quelconque réalisme de leur analyse.

Amartya Sen affirme qu'avec ses deux grandes œuvres Adam Smith est l'un des premiers et peut-être le dernier (avec Keynes) à avoir réussi à construire une théorie économique à la fois réaliste et rationnelle, à avoir synthétisé savoir relatif et savoir absolu. Toute la brillante carrière de théoricien et de philosophe de Sen

${ }^{10}$ La contrainte forcée par l'instrument mathématique sur la démarche théorique des économistes est un problème largement sous-estimé et, au demeurant, fort peu étudié.

${ }^{11}$ Aujourd'hui postulée sans difficulté, la continuité des fonctions d'utilité fera passer d'innombrables nuits blanches à Pareto qui constatant leurs discontinuités dans le monde 'réel' cherchait désespérément une solution mathématique impossible pour conserver cette caractéristique du comportement des agents. Une fonction d'utilité continue 'idéalise et renforce' (pour des raisons mathématiques) l'axiome initial mais que reste-t-il de l'intuition 'réaliste' initiale?

${ }^{12}$ Par exemple, la résolution formelle par Wald, dans les années 1930, de la problématique des prix et des quantités négatives négligées par Walras, Pareto et Cassel ou même l'apparition des théorèmes de point fixe dans les années 1950 sont de belles illustrations de la généralisation, et donc de la cumulativité, des résultats formels obtenus en termes de théorèmes d'existence. 
n'est rien d'autre qu'une très longue tentative de réconciliation entre ces deux origines de la science économique: la science économique comme éthique et la science économique comme 'engineering'. La première remonte à Aristote et la seconde (injustement!) à Walras. L'importance démesurée donnée par les économistes à la rationalité aprioriste d'une théorie économique 'de l'ingénieur' s'est faite au détriment des sentiments moraux pour utiliser la terminologie smithienne: self-interest et rationality doivent faire place à une pluralité des motivations. Comment le dire plus succinctement que Sen lui-même:

The professor of moral philosophy and the pioneer economist did not... lead a life of spectacular schizophrenia. Indeed, it is precisely the narrowing of the broad Smithian view of human beings, in modern economics [started unwillingly by Walras], that can be seen as one of the major deficiencies of contemporary economic theory (1987, p. 28).

Quelles sont alors les conséquences d'une telle opposition, ou plutôt d'un tel biais en faveur de la science économique considérée comme engineering economics, sur la cumulativité du savoir en économie.

\section{CUMULATIVITÉ QUANTITATIVE ET CUMULATIVITÉ QUALITATIVE: UN PROGRÈS THÉORIQUE SAANS PROGRÈS EMPIRIQUE EST-IL UN PROGRÈS SCIENTIFIQUE?}

En dépit de multiples efforts, il est difficile de trouver des références explicites $^{13}$ chez les économistes anciens et modernes à la notion de cumulativité du savoir. Soit le sujet ne les intéresse pas, soit ils ne se posent même pas la question: la proximité supposée des sciences dures rendrait cette cumulativité évidente. Pour la plupart des théoriciens (une fois encore dans le sens particulier où l'entendent les économistes) et des économètres, l'existence d'une cumulativité du savoir est trop évidente pour nécessiter même une brève discussion en dépit du rôle qu'un tel concept joue dans notre profession. L'auteur n'a-t-il pas entendu récemment l'un de ses collègues affirmer tout de go qu'il ne comprenait pas cette interrogation car, comme 'nous sommes d'accord sur les instruments d'analyse', cette question devenait sans objet... Notre discipline a développé un tel stock de techniques analytiques que nous sommes passés dans une autre catégorie scientifique que la philosophie, les sciences politiques, l'histoire et bien sûr, horresco referens, la sociologie. Dans ces disciplines immatures, argumentent les tenants du mainstream, vous continuez à vous disputer sans fin sur les mêmes questions fondamentales. C'est en raison par exemple de l'absence de cumulativité des connaissances en philosophie politique qu'il est encore utile pour les politologues de relire Hobbes, Locke, Rousseau ou même Rawls et Nozick. La science économique, prétendra le même économiste, a dépassé ce stade. L'application de méthodes mathématiques aurait transformé la matière en discipline cumulative dans laquelle le progrès est constant et évident. Il suffirait donc de ne lire que la littérature la plus récente et d'ignorer tout ce qui a plus de cinq ans d'âge. Quatre-

\footnotetext{
${ }^{13}$ Voir par exemple Keynes (1921, Part III), Hey (1983, p. 1) ou Backhouse (1997, p. 4).
} 
vingt-dix-neuf pour cent des économistes de moins de 40 ans n'ont pas lu Keynes, ne connaissent de Walras que sa pseudo loi, de Pareto un optimum sans grand rapport avec l'original et pensent que la théorie de la productivité marginale est une invention de Ricardo. Pire encore, dans le domaine de la théorie monétaire, les classiques des années 1970, comme Friedman, Tobin ou même Lucas ne sont cités que de seconde main ou par ouï dire. Pigou à qui l'on demandait dans les années 1940 d'écrire la recension d'une réédition d'auteurs classiques répondait déjà qu'il n'avait pas de temps à consacrer à l'étude des théories erronées d'auteurs morts depuis plus d'un siècle.

Les choses, nous l'avons vu, ne sont pas si simples: 'Knowledge does not merely accumulate in a straigthforward or piecemeal fashion' (Lawson, 1987, p. 967). L'accumulation du savoir en sciences économiques ne peut être un simple processus quantitatif. L'idée que le développement d'une discipline scientifique n'est qu'un processus d'accrétion linéaire par le biais d'une simple addition d'éléments ajoutés séparément, ou par combinaison, à un stock toujours croissant de connaissance a clairement été mise en doute par Kuhn, il y a plus de trente ans (1970, p. 1). Au cours d'une phase 'quantitative normale' de développement d'une discipline, Kuhn montre bien que les anomalies et les problèmes non résolus se multiplient apportant la discipline à une situation de crise qui provoque alors un changement qualitatif. Et les exemples de tels changements qualitatifs sont relativement fréquents en science économique: la montée rapide et la chute non moins brutale du modèle keynésien; le relatif abandon récent de l'équilibre général au profit de la théorie des jeux, les changements constants de doctrine en politique monétaire, le retour des imperfections avec les modèles nouveauxkeynésiens, etc... Toutefois, cette hypothèse de changements qualitatifs relativement fréquents n'implique pas que ces modifications soient le reflet de retournements théoriques complets ou arbitraires. La continuité théorique du noyau dur domine clairement les discontinuités et les changements qualitatifs qui ont lieu dans la ceinture protectrice de la science économique. On doit néanmoins admettre que la cumulativité du savoir des économistes exhibe des moments quantitatifs et qualitatifs.

Tout cela nous ramène naturellement et finalement à la question cruciale du rapport entre formalisation et observation, aux rapports difficiles entre théorie économique et économétrie (à supposer que l'économétrie s'intéresse encore au 'monde réel'). Et c'est au niveau de cette interaction problématique que se joue, en dernière analyse, la difficile cumulativité du savoir en science économique. Cette discussion devrait nous amener à deux conclusions peu surprenantes. D'une part, en donnant une priorité évidente à la formalisation théorique sur l'observation empirique (même formalisée), les économistes ne respectent pas la méthodologie poppérienne dont la majorité d'entre eux se réclame pourtant avec force. D'autre part, et en dépit d'expériences récentes, les économistes sont peu disposés à abandonner le confort de leur paradigme du choix rationnel sur lequel repose leur identité scientifique. La réalisation d'un partage cumulatif suggéré par Denise Pumain semble pour le moins problématique...

En forçant à peine le trait, l'opposition-interaction entre formalisation et observation peut être résumée pour les économistes d'une manière très simple, voire simpliste. La théorie économique (mathématisée à 95\%) analyse la foultitude de problèmes économiques observées dans la réalité au moyen de modèles 
formels tous basés sur la matrice de la théorie du choix rationnel individuel. Les individus sont pratiquement tous modélisés comme des agents optimisateurs (i.e. résolvant à l'exemple du théoricien un programme d'optimisation sous contrainte); ils se meuvent dans un environnement très étroitement et précisément définis (concurrence parfaite, concurrence monopolistique, jeux coopératifs ou non, etc.). Pour simplifier même les choses, l'économiste n'hésite pas à représenter une société composée d'une multitude d'individus par un seul agent représentatif à durée de vie illimitée.

En complément à cette démarche qui, selon Hicks, permet à l'économiste d'avoir 'a good intellectual game', on trouve une autre démarche formelle, l'économétrie (l'observation est donc elle aussi formalisée). Ici, l'économètre construit une théorie de l'analyse des séries statistiques qui conduit à une estimation formelle de relations quantitatives entre variables. Ce travail se fait sous l'hypothèse que ces données statistiques peuvent être modélisées comme étant le résultat de distributions probabilistes sous-jacentes.

Ces deux composantes de la science économique ont naturellement beaucoup de points communs (notamment l'usage de méthodes mathématiques très sophistiquées). Elles devraient normalement se renforcer l'une l'autre. Or, il n'en est rien. En particulier, la démarche théorique initiale est, la plupart du temps, complètement déconnectée du test formel de sa validité, et vice-versa! Hendry va même jusqu'à affirmer que le gros du travail des économètres consiste à estimer et non pas à tester des modèles théoriques. L'intégration logique de ces deux composantes telle qu'elle était envisagée par les économistes des années 1950-60 est beaucoup moins assurée aujourd'hui qu'on ne l'espérait, ou pire que l'on feint encore de le croire aujourd'hui. Les avatars récents de la calibration des modèles d'équilibre général et des computational experiments illustrent en fait que l'écart entre les deux grandes composantes de la science économique semble se creuser.

Compte tenu de son succès foudroyant (et de la nouvelle relation qu'elle établit entre théorie et 'estimation'), la méthodologie des équilibres généraux calculables vaut la peine que l'on s'y arrête un instant. En effet, même si cette approche trouve son origine chez Kydland et Prescott et leur théorie des cycles réels, elle s'est maintenant répandue dans une kyrielle d'autres domaines. Explicitement cette approche affirme que le choix d'une théorie ne dépend pas de sa capacité à refléter au mieux les données statistiques disponibles (les modèles économétriques ne sont en effet pour eux pas capables d'estimer des paramètres structuraux invariants par exemple à des changements de politique économique). La validité d'une théorie ne peut donc pas être testée au moyen de méthodes économétriques (et donc explicitement historique). En d'autres termes, pour Kydland et Prescott, un modèle théorique n'est pas (plus!) un ensemble d'hypothèses sur le monde réel, mais un simple instrument heuristique qu'il faut 'calibrer' (et non plus 'estimer' la valeur des paramètres puisque c'est impossible). Par 'calibrer', ces deux auteurs entendent simplement 'choisir' la forme fonctionnelle et la valeur arithmétique de paramètres, de manière à ce que le modèle théorique 'singe' au mieux le monde réel (exprimé par des séries statistiques). La méthodologie walrasienne selon laquelle 'la théorie pure n'atteint aucune confirmation de la réalité' atteint son stade ultime. Dans leur démarche pionnière sur les cycles réels, Kydland et Prescott utilisent le modèle de croissance néo-classique standard, le 'calibre' en sélectionnant des formes fonctionnelles, et en donnant des 
valeurs numériques aux paramètres des fonctions d'utilité et de production, de manière à ce que le modèle donne l'impression d'expliquer les facteurs de la croissance économique de longue période des Etats-Unis (en particulier les chocs technologiques). Le modèle est à la fois hautement formalisé dans son énoncé et informel dans la méthode de choix des valeurs données aux paramètres. En bref, l'un des courants théoriques actuellement dominants renouvelle la procédure de divorce entre formalisation et observation d'une manière différente de celles de Walras ou de Debreu. La théorie n'est qu'un instrument et non une description, même stylisée de la réalité; par nécessité la théorie est une abstraction nécessairement fausse et donc non économétriquement estimable; la méthode de calibration permet alors de rapprocher la théorie économique de sciences dures dans lesquelles, par exemple, le scientifique formate, 'calibre', son thermomètre pour être certain qu'il marque $100^{\circ} \mathrm{C}$ lorsque l'eau bout et $0^{\circ}$ lorsqu'elle gèle... En toute rigueur méthodologique, la comparaison devrait s'arrêter là: le thermomètre est strictement un instrument passif de mesure de la température; il ne peut prévoir en rien les variations futures de la température. Les modèles d'équilibre stochastique calculables sont en revanche dangereusement plus ambitieux: ils prétendent en effet être utilisés pour formuler des prévisions... Une fois encore, le gouffre entre théorie et observation est béant; mais vouloir le combler à tout prix pourrait s'avérer dangereux.

\section{REMARQUES CONCLUSIVES ET PROVISOIRES : DIVORCES INTERNE ET EXTERNE ET CONTRIBUTION FONDAMENTALE}

En guise de conclusions extrêmement provisoires à ce qui vient d'être suggéré, il convient de revenir dans un premier temps sur ce divorce interne à la science économique entre théorie et observation; dans un second temps, on tente d'expliquer pourquoi ce divorce interne influe d'une manière décisive sur le divorce 'externe' d'avec les autres sciences sociales; et donc finalement, pourquoi le partage cumulatif interdisciplinaire proposé par Denise Pumain a peu de chance, en dépit de récents clins d'œil à la psychologie expérimentale (Kahneman) et à la géographie (Krugman) d'obtenir un accueil autre que glacialement poli de la part des économistes.

Le divorce interne qui semble déchirer la science économique entre théorie et économétrie pose en fait d'une manière plus complexe que prévu l'idée de progrès et de cumulativité des connaissances. Blaug (1994) établit volontiers une distinction entre progrès théorique et progrès empirique (même si formalisation et observation sont tous deux comme on l'a vu hautement ... formalisées). Les progrès théoriques cumulables sont purement heuristiques: ils garantissent une plus grande clarté conceptuelle et fournissent des innovations analytiques. Parmi ces progrès théoriques, on peut découvrir des concepts permettant un accroissement de la généralité, de la précision, de la rigueur des modèles, l'élimination d'erreurs, d'incohérences et l'amélioration de leur simplicité (de leur beauté esthétique) et de leur capacité prévisionnelle. Les progrès empiriques cumulables sont évidemment beaucoup plus flous que les progrès théoriques. Blaug les définit comme 'a deeper grasp of the inner springs of economic behaviour and hence of the opera- 
tions of the economic system' (1994, pp. 116-17) ${ }^{14}$. Cette approche est ensuite liée à l'idée discutée plus haut qu'il est nécessaire de combiner ces deux types de progrès de manière à pouvoir élaborer des prévisions quantitatives et qualitatives sur l'évolution d'un système économique. La part relative de chacun de ces types de progrès cumulables est évidemment discutable. Néanmoins, et l'examen de n'importe quel numéro récent d'une grande revue économique confirmera sans peine que, dans le divorce formalisation-observation, le 'conjoint' théorique occupe une position dominante. Ce déséquilibre est particulièrement bien illustré par les coefficients plus élevés accordés par exemple dans les critères d'évaluation académiques des départements d'économie britanniques aux articles purement théoriques au détriment des contributions d'économie appliquée. Cette inégalité nous amène naturellement à poser la question de savoir s'il y a progrès de la connaissance (et donc possibilité de cumulativité) en sciences économiques si la majeure partie des progrès se situe strictement dans le domaine théorique. $\mathrm{Ou}$, en d'autres termes, un progrès strictement théorique non lié à un progrès empirique est-il vraiment un progrès cumulable en science économique?

Il faudrait naturellement consacrer un article entier à cette question cruciale qui impliquerait notamment que l'on s'accorde sur la capacité d'observations empiriques à résoudre des problèmes théoriques et à faire ainsi progresser théorie et observation. Obnubilés par la modélisation théorique, la plupart des économistes seraient probablement d'accord pour admettre qu'un progrès théorique qui ne contribuerait en rien à un progrès empirique peut néanmoins être considéré comme une addition à leur stock de connaissances. On peut néanmoins sérieusement douter qu'un progrès strictement théorique (la découverte par exemple dans les années 1950-60 de différents théorèmes de point fixe) fasse progresser la compréhension de la logique de fonctionnement du monde économique.

Cette domination de la modélisation théorique sur l'observation si répandue chez les économistes trouve probablement son explication dans le fait que, pour beaucoup d'entre-nous, les modèles théoriques jouent le rôle de l'expérience, qu'ils en prennent la place. Pour renverser une remarque de Clower, on pourrait dire que pour les économistes mainstream, le modèle est le message alors que clairement le message, comme pour Walras et contrairement à Debreu, devrait toujours être plus que le modèle (1984, p. 264). Ne donnons-nous pas paradoxalement la priorité à une théorie économique cumulable - et donc plus proche des sciences dures - parce que, contrairement aux sciences dures, nous sommes dans l'impossibilité de pratiquer une 'vraie' expérimentation? En parlant de l'usage que fait Lucas de la théorie de l'équilibre général, Townsend (1988, p. 5) affirme que 'les modèles d'équilibre général peuvent être considérés comme des laboratoires dans lesquels le chercheur mène des expériences'. Devant l'extrême complexité des économies réelles, le seul moyen de réfléchir sur cette complexité est bien de réfléchir sur un modèle de l'économie réelle (qui forcément n'est pas un modèle à l'échelle $1: 1$ et qui, par définition n'est pas non plus réfutable au sens de Popper. Considérer des sentiers de prix et de quantités stochastiques comme des points d'équilibre est devenu complètement admis par les théoriciens

${ }^{14}$ En d'autres termes, exactement ce que, aux Etats-Unis, les doctorants trouvent inutiles pour mener une carrière académique. 
modernes. Du même coup, on postule comme résolu le problème de la stabilité dynamique d'une économie de marché au centre des préoccupations de la théorie économique pendant près de deux siècles; et la problématique keynésienne devient elle aussi 'irrelevante' pour la théorie à défaut de l'être pour le chômeur au coin de la rue. Est-ce finalement très différent de ce répétait Debreu en 1991 lorsque dans sa Presidential Address à l'American Economic Association, il affirmait que la rigueur de la déduction logique doit servir de substitut à une base expérimentale défaillante. Avait-il vraiment tort?

Le divorce externe d'avec les disciplines voisines découle naturellement de ce divorce interne. Depuis plus d'un siècle, et dans la poursuite d'une mythique scientificité, les économistes ont tout tenté pour se distancer des autres sciences humaines. Par exemple, dans le cadre de la théorie du choix rationnel, de Pareto aux préférences révélées de Samuelson, les économistes n'auront cesse de déconnecter leur théorie du comportement du consommateur de tout psychologisme (du type loi de Fechner) et de toute introspection. La théorie pure est même considérée par certains comme le moyen par excellence de bannir l'introspection et la subjectivité du raisonnement des économistes. Ce n'est que très récemment que, sous le couvert de la microéconomie empirique moderne, un économiste comme Vernon Smith et un psychologue comme Daniel Kahneman tentent un retour à l'observation pour tester (sans grand succès d'ailleurs) les fondements de la théorie du consommateur sur des cohortes d'étudiants ${ }^{15}$. Toutefois, et en contrepoint, la nouvelle géographie économique utilise l'artillerie lourde de la formalisation des économistes non pour tendre la main aux géographes mais pour redécouvrir par le biais de la formalisation des résultats dont certains sont connus depuis bien longtemps. À croire que ce qui n'est pas formalisé n'existe pas !

Même si ces récentes tentatives (même couronnées par un Prix Nobel) semblent annoncer un retour à une approche plus réaliste et à l'expérimentation empirique, nous sommes encore loin d'une conversion. Tant s'en faut. Les priorités de la science économique sont plus que jamais fermement ancrées dans la formalisation théorique. Une étude récente montre que, dans les grandes revues scientifiques, la proportion des articles d'économie appliquée est le plus faible des sciences humaines mais aussi et surtout plus faible que dans des disciplines dites 'dures' du type chimie et, c'est un comble, de la physique tant enviée. L'identité même de la science économique, le sentiment d'appartenance des économistes à une communauté scientifique et leur différenciation par rapport aux autres sciences humaines reposent essentiellement sur cette capacité de modélisation formelle et de priorité donnée à la théorie sur l'observation. De plus, et là comme ailleurs, les économistes doivent tous plaider coupables, la recherche théorique s'est largement détachée de la politique économique. Même si le discours économique domine largement aujourd'hui le débat politique, rarement (et paradoxalement) dans l'histoire de la science économique, les théoriciens ont été aussi peu influents sur la conduite de la politique économique. Jamais les déficits budgétaires (sciemment recherchés par certains gouvernements comme l'administration

${ }^{15}$ Dans un article récent, Pieters et Baumgartner (2002) démontrent i) l'extrême pauvreté des rapports entre psychologues et économistes (p. 504) et ii) le fait, que les autres sciences humaines citent plus volontiers les économistes que les économistes ne se réfèrent aux disciplines voisines (p. 497-502). 
Bush) n'ont été plus importants; jamais les taux de croissance de la dette publique (y compris en Suisse) n'ont été aussi élevés et pourtant, au niveau théorique, il existe un large consensus sur la nécessité d'un retour à l'équilibre des finances publiques. Plus aucun chercheur américain ne prône le deficit spending alors que l'Etat fédéral américain n'a jamais connu en temps de paix des écarts pareils entre recettes et dépenses. À quoi servons-nous donc? Pourquoi après trente années d'économistes rois, ne sommes-nous plus en mesure d'influer la politique économique alors que, paradoxalement, l'idéologie du discours économique néo-libéral dominant emprunte largement, mais pas toujours sagement, des pans entiers de ses arguments à une théorie économique à moitié digérée?

Finalement, que dire d'autre et de plus constructif à propos de la transférabilité-cumulativité des concepts entre les diverses disciplines des sciences humaines?

L'idée de partir d'un point de vue strictement disciplinaire pour 'communiquer aux autres un résultat fondamental de [s]a discipline' est naturellement très séduisant. Et pour cause... voilà à nouveau l'éconoiste victime consentante de l'impérialisme de la théorie économique. L'économiste pourrait par exemple communiquer et illustrer utilement l'acquis fondamental de la théorie économique qu'est l'idée de coordination marchande par les prix. Comment pourrionsnous alors élargir ce résultat formel et hautement sophistiqué à l'extrême complexité des autres disciplines qui tentent aussi, et d'une manière plus relativiste, d'expliquer les phénomènes de coordination entre individus. L'idée centrale de la main invisible fait appel au moins depuis Smith à des principes de philosophie politique, de psychologie, de théorie du comportement de l'agent évoluant dans un environnement spatial, au respect de la rule of law et de la sainteté des contrats, à la pluralité des motivations et des critères décisionnels liée à des phénomènes culturels, etc... En d'autres termes, et foin de modestie, quittant les marges disciplinaires 'négociables', ne faudrait-il pas retourner à l'une des constantes les plus structurées et les plus fondamentales de l'une de nos disciplines pour ensuite, par accrétion, par cercle concentrique, la complexifier en faisant appel à l'ensemble des autres sciences sociales? On pense immédiatement - mais n'est-ce pas une tarte à la crème - à la théorie du choix rationnel...!

Tout cela n'est guère original et nécessiterait un travail en commun qui tiendrait du programme de recherche de très longue haleine. Deux citations classiques et bien connues faisaient déjà écho à ces préoccupations, l'une en 1803 et l'autre en 1909. L'une est de Sismondi et l'autre de Pareto, tous deux des économistes pourtant complètement sceptiques sur la valeur absolue de la théorie économique.

L'économiste... qui préconise une loi en ne prenant en considération que ses seuls effets économiques n'est pas trop théoricien; il ne l'est pas assez puisqu'il néglige d'autres théories qu'il devrait réunir à la sienne pour porter un jugement sur [un] cas pratique... Il serait peu raisonnable de prétendre régler les phénomènes économiques par les seules théories de l'économie pure. (Pareto, 1909, pp. 20 et 248)

Les sciences humaines sont comme des secteurs de cercles concentriques dont le nombre est infini; l' homme est placé à leur centre, il voit entre chaque rayon une science; il découvre ainsi l'enchaînement et les rapports des unes avec les autres, mais plus la science s'éloigne de sa vue et de sa porté, plus elle s'élargit, plus elle s'étend; il a beau la diviser et la subdiviser, chacune de ses portions est illimitée, et fait partie de l'infini. (Sismondi, 1803, I, p. XIX) 
Sismondi ne propose-t-il pas là un superbe programme de recherche d'épistémologie des sciences humaines? Ne reste-t-il pas 'simplement' à choisir le point de départ de l'un de ces rayons qui traversent les cercles concentriques des diverses sciences humaines? N'y a-t-il pas là finalement une occasion unique de cumulativité 'à l'infini'? Mais les économistes le veulent-ils vraiment?

\section{Centre d'études interdisciplinaires Walras-Pareto \\ Université de Lausanne}

\section{RÉFÉRENCES BIBLIOGRAPHIQUES}

Backhouse, R. (1997). Truth and Progress in Economic Knowledge, Cheltenham: Edward Elgar.

Baranzini, R. et Bridel, P. (2004). «L'Ecole de Lausanne, l'utilité marginale moyenne et l'idée de marché », Actes du Colloque Charles Gide, Grenoble, 2003, à paraître.

Blaug, M. (1980). The Methodology of Economics, Cambridge: Cambridge University Press.

Blaug, M. (1994). «Why I am not a Constructivist: Confessions of an Unrepentant Popperian » in New Directions in Economic Methodology (R.E. Backhouse, éd.), London and New York: Routledge, pp. 109-36.

Bourdieu, P. (1998). Contre-feux, Paris: Liber-Raisons d'Agir.

Bridel, P. (2002). «The Endogeneity of Money: Walras and the 'Moderns'», in Exogeneity and Endogeneity: The Quantity Theory of Money in the History of Economic Thought and in Modern Policy (B. Schefold, éd.), Marburg, Metropolis, pp. 227-47.

Clower, R. (1984). «Afterword» in Money and Markets. Essays by Robert Clower (D. Walker, éd.), Cambridge: Cambridge University Press, 1984, pp. 259-272.

Debreu, G. (1966). Théorie de la valeur: analyse axiomatique de l'équilibre général, Paris: Dunod.

Debreu, G. (1991). «The Mathematization of Economics», American Economic Review, 81, pp. 1-7.

Friedman, M. (1953). «The Methodology of Positive Economics», Essays in Positive Economics, Chicago: University of Chicago Press, pp. 3-46.

Hahn, F.H. (1985). «In Praise of Economic Theory», in Money, Growth and Stability, Cambridge, MIT Press, pp. 10-28.

Hempel, C.G. (1966). Philosophy of Natural Science, Englewoods Cliffs : Prentice-Hall.

Hey, J. (1983). «Whither Uncertainty?», Economic Journal, 91, pp. 130-39.

Ingrao, B. and Israel, G. (1990). The Invisible Hand. Economic Equilibrium in the History of Science, London: MIT Press.

Keynes, J.M. (1921). A Treatise on Probability, in Collected Writings of John Maynard Keynes, vol. VIII, Cambridge, Cambridge University Press, 1973.

Kuhn, T. (1970). The Structure of Scientific Revolution, Chicago: Chicago University Press.

Laudan, L. (1984). Science and Value, Berkeley, Los Angeles and London: University of California Press.

Lawson, T. (1987). «The Relative/Absolute Nature of Knowledge and Economic Analysis», Economic Journal, 97, pp. 951-70.

Malinvaud, E. (1995). «L'économie s'est rapprochée des sciences dures, movement irréversible mais achevé» in L'économie devient-elle une science dure? édité par A. d'Autume et Jean Cartelier, Paris: Economica, pp. 9-17.

Pareto, V. (1909). Manuel d'économie politique, Paris: Giard et Brière.

Passeron, J.-C. (1991). Le raisonnement sociologique. L'espace non-poppérien du raisonnement naturel. Paris: Nathan. 
Pieters, et Baumgartner, (2002). «Who talks to Whom? Intra- and Interdisciplinary Communication of Economic Journals », Journal of Economic Literature, 40, pp. 483-509.

Pumain, D. (2005). «Cumulativité des connaissances. Texte introductif», Revue européenne des sciences sociales, 43 , pp. 5-12.

Rabin, M. (1998). «Psychology and Economic Theory», Journal of Economic Literature, 36, pp. 1146.

Sargent, Th. Et Velde F. (2002). The Big Problem of Small Change, Princeton et Oxford, Princeton University Press.

Sen, A. (1987). On Ethics and Economics, Oxford: Basil Blackwell.

Schumpeter, J.A. (1954). History of Economic Analysis, London: Oxford University Press.

Sismondi, J.C.L. Simonde de. (1803). De la richesse commerciale, Genève: Paschoud, 2 volumes.

Smith, A. (1776). An Inquiry into the Nature and Causes of the Wealth of Nations (R.H. Campbell et A.S. Skinner, éd.), 1976, Oxford: Clarendon Press, 2 volumes.

Townsend, R. M. (1988). «Models as Economies», Economic Journal, 98, Conference Papers, pp. 124.

Weintraub, E.R. (1985). General Equilibrium Analysis; Studies in Appraisal, London: Cambridge University Press.

Walras, L. (1874-77). Eléments d'économie politique pure, Auguste et Léon Walras - Euvres économiques complètes, vol. VIII, Paris: Economica, 1988. 